\title{
Z-Score loreta neurofeedback as a potential therapy in cognitive dysfunction and dementia
}

\begin{abstract}
Introduction of QEEG/LORETA electrical brain imaging has improved our diagnostic ability in neuropsychiatric practice by enhancing identification of dysregulated cortical areas implicated in patient symptoms. Additional use of LORETA Z-score neuro feedback (NFB) enables us to directly target these areas of dysregulation in order to improve associated symptoms. Based on the review of 250 patients treated in our clinic suffering from neuropsychiatric illness and treated with Z-score LORETA NFB, analysis of cases of cognitive dysfunction and dementia are presented. Specific areas of dysregulation attributed to particular conditions identified by LORETA are discussed. Follow up findings of QEEG/ LORETA electrical imaging after NFB therapy (including computerized cognitive testing results) are shown. This paper summarizes my experience with LORETA Z-score NFB as a tool for therapy of cognitive dysfunction. In addition, this form of NFB is able to improve cognitive functions of individuals suffering from memory, information processing and other cognitive dysfunctions. Extensive presentations of selected cases are used for demonstration of results from my practice. Twenty five out of 35 patients $(71 \%)$ who suffered from static cognitive dysfunction were identified as having an objective improvement (on average 10 points through cognitive testing) with NFB therapy. In addition, the subjective cognitive improvement and an objective reduction of QEEG abnormalities with NFB were also achieved in most of the patients. These results are very promising and indicate good effectiveness of LORETA Z-score NFB as cognitive enhancement tool.
\end{abstract}

Keywords: z-score, loreta, neurofeedback, eeg, qeeg, cognitive enhancement, dementia
Volume I Issue 6 - 2014

Lucas Koberda J

Tallahassee Neuro-Balance Center, USA

Correspondence: Lucas Koberda J, Professor of Neurology, Tallahassee Neuro-Balance Center, 4838 Kerry Forest Parkway, Tallahassee, FL 32309, USA, Tel 850-459-8263, Email JLkoberda@yahoo.com

Received: May 27, 2014 | Published: November 12, 2014

\section{Introduction}

Alzheimer's disease (AD) is the most common form of dementia. It is a neurodegenerative disease characterized by gradual loss of mental ability, severe enough to interfere with normal activities of daily living. AD typically occurs in elderly age, and is marked by a decline in cognitive abilities such as remembering, reasoning, and planning. A person with $\mathrm{AD}$ exhibits a gradual decline in mental functions, often beginning with slight memory loss, followed by losses in the ability to maintain employment, to plan and execute familiar tasks of daily living, and to reason and exercise judgment. $\mathrm{AD}$ usually becomes symptomatic after age 60. However, the histopathological abnormalities involving amyloid formation are likely to start many years before the disease manifestation. After age 80 , close to $50 \%$ of individuals display clinical symptoms of this disease. In Florida, it is estimated that the number of individuals with $\mathrm{AD}$ is close to 500,000 . There is currently no effective treatment of AD. Medications available on the market have only mild symptomatic benefits, with frequently significant side effects. A typical MRI of the brain of $\mathrm{AD}$ patients shows brain atrophy that is most pronounced in the temporal and frontal areas. A recently FDA-approved PET scan (amyloid imaging) generally shows increase in amyloid deposits in individuals affected by this disease. QEEG frequently reveals increase in delta and theta frontal and temporal power. ${ }^{1}$ Neurofeedback has been shown previously to be helpful in selected cases in memory and cognitive enhancement. ${ }^{2-7}$ However, our experience with patients suffering from AD is still very limited. Therefore, I would like to share my experience describing testing and results of NFB therapy in five $\mathrm{AD}$ patients from my practice.

NFB has also been underestimated in the clinical arena as a potential therapeutic modality. Standard one or two electrode NFB has been reported as beneficial in relieving depression symptoms in several investigations, including a randomized controlled study. ${ }^{8} \mathrm{NFB}$ training had profound effects on emotion and cognition. The authors replicated earlier findings that enhancing the left frontal activity led to alleviation of depressive symptoms. Moreover, cognitive tests revealed that the asymmetry training improved performance of executive function tests, whereas the placebo treatment did not show improvement. $^{8}$

In Z-Score NFB, a real-time comparison to an age-matched population of healthy subjects is used for data acquisition, simplifying protocol generation and allowing clinicians to target modules and hubs that indicate dysregulation and instability in networks related to symptoms. Z-score NFB increases specificity in operant conditioning, providing a guide that links extreme Z-score outliers to symptoms, and then reinforcing $Z$-score shifts toward states of greater homeostasis and stability. The goal is increased efficiency of information processing in brain networks related to the patient's symptoms. ${ }^{9}$

A recently introduced method called Low Resolution Electromagnetic Tomography (LORETA) Z-score NFB is capable of targeting specific dysregulated anatomical structures, many of which are in deeper cortical locations. ${ }^{9-10}$ For example, the Insula and Anterior Cingulate have been identified as potential NFB target sites to improve pain control in patients who display electrical dysregulation of these areas. ${ }^{10}$

I would like to focus on a few of our patients who successfully completed Z-score LORETA NFB with marked improvement in cognitive function. Cognitive function, which is often impaired in patients with depression, usually improves after NFB therapy. What is also important to note that NFB seems to be not only effective in patients with static (non-progressive) cognitive dysfunction but also with progressive neurodegenerative disorder like AD. 


\section{Materials and methods}

Deymed Truscan 32 (Deymed Diagnostic, Payette, ID) EEG equipment was combined with Neuroguide (Applied Neuroscience, Inc.) software. The NFB protocol included surface and LORETA (NF1/NFB2) feedback, in an alternating protocol while focusing on a symptom checklist including: attention deficits, concentration problems, executive function problems and short-term memory. NFB sessions were conducted with a frequency of once to twice per week, using auditory or auditory and visual feedback combined.

A commercially available computerized neurocognitive testing battery was used for the initial assessment of many patients (NeuroTrax Corp, Bellaire, TX). NeuroTrax Corporation cognitive testing is a computerized neuropsychological assessment where the patient is compared to aged and education matched healthy controls where mean is 100 and the standard deviation is 15 . NeuroTrax (Mind Streams) computerized cognitive testing has been previously extensively tested by its manufacturer for reliability. To minimize learning across sessions, three alternative forms of cognitive tests were developed with identical psychometric properties but different items. Equivalence of all three alternate forms was demonstrated to have an acceptable test-retest reliability coefficient as well as the alternate form reliability coefficients. QEEG analysis was completed using commercially available Neuroguide software (Applied Neuroscience, Inc.) and previously recorded 19 channel digital EEG.

Approximately 1-3 minutes of artifact-free eyes closed EEG segments were selected after previously recording EEG with the Deymed, Truscan 32, (Deymed Diagnostic, Payette, ID) and subjected to further QEEG analysis. NFB therapy consisted of 30-minute sessions once or twice a week using auditory and/or visual feedback.

\section{Results}

\section{Case I}

40-year-old female was referred to me by psychiatrist who was previously treated for major depression and did not respond to pharmacological treatment. Prior to NFB, she was treated with Electro-Convulsive Therapy (ECT), which was not successful in relieving her depression. Instead, the individual sustained major memory impairment and visual-spatial difficulties. Since she was not responding to conventional therapy, her psychiatrist referred her to my practice for NFB therapy. The patient's cognitive and depressive dysfunction caused inability to continue her employment as a pharmaceutical representative. Initial LORETA showed several areas of electrical dysregulation including (Figure 1A) BA-5 (secondary sensorimotor cortex), BA-9 (prefrontal cortex) and temporal cortex (not shown).

Her computerized cognitive testing before NFB showed deficiency in memory, information processing speed and visual-spatial domains. After 10 sessions of Z-score LORETA NFB, the patient reported major improvement in her mood (as well as mild memory improvement). Repeated computerized cognitive testing revealed a marked improvement of previously deficient cognitive domains (Figure 2). Memory score increased from 85.4 to 102.8, information processing speed rose from 90.8 to 97.7 and visual-spatial domain went up from 80.8 to 100.7. In addition, post NFB LORETA showed an improvement of previously identified electrical dysregulation (Figure 1B). After successful NFB therapy, the patient also was able to come back to gainful employment in the medical field (after few years of being unemployed).

\section{Case 2}

58year old university professor was seen in my office due to major depression associated with anxiety and memory problems. She noticed problems with teaching due to possible cognitive dysfunction. (Figure 3) shows her LORETA imaging before NFB, which identified several areas of electrical dysregulation including the cingulate cortex and left temporal region.

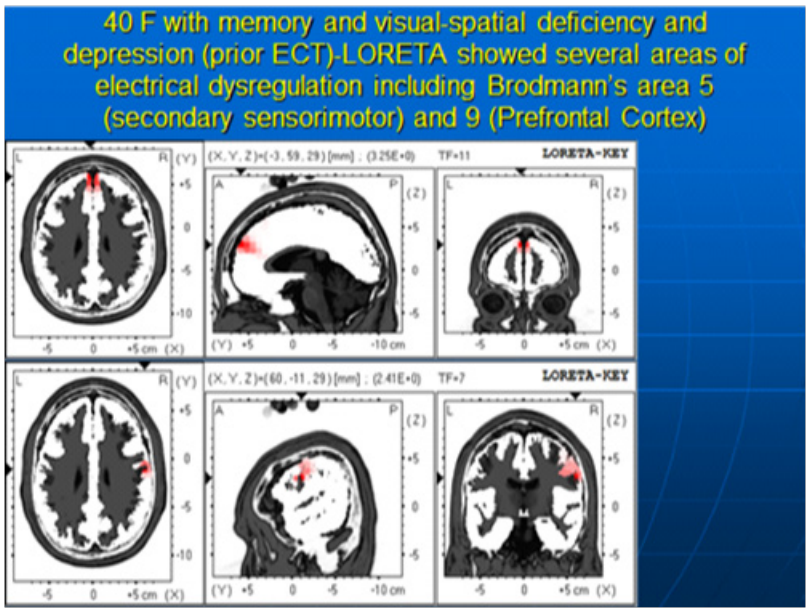

Figure I a Pre-NFB LORETA of 40 year old female diagnosed with depression associated with cognitive dysfunction. Areas of cortical dysregulation are shown in red.
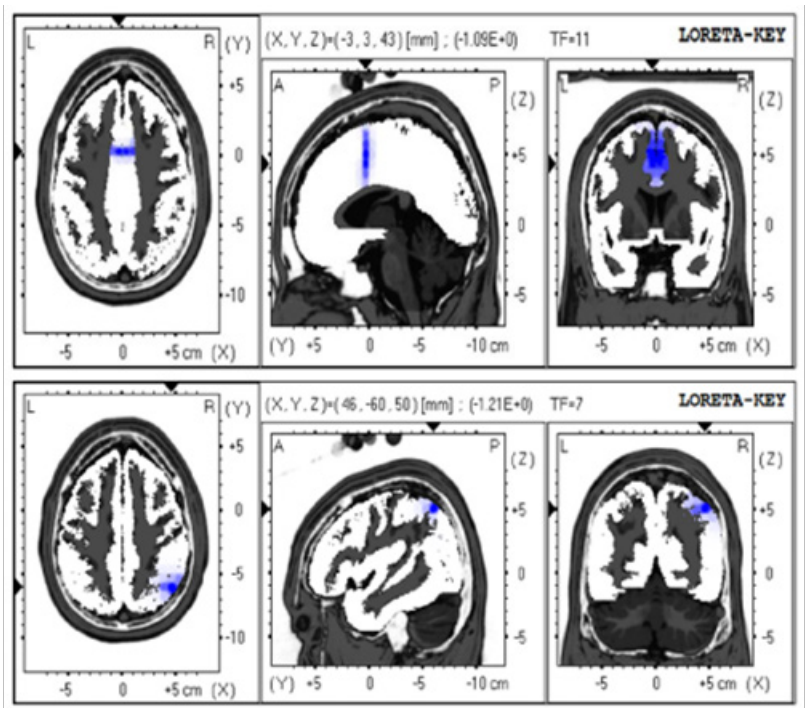

Figure Ib 40 year old female-LORETA after NFB-shows resolution of previously electrically dysregulated BA-5 and BA-9.

The computerized cognitive testing completed before NFB identified deficiency of memory and executive function. Blood work was negative for B12 deficiency. After 10 sessions of NFB, major improvement in memory (from 42.8 to 107.1) was recorded on neurocognitive testing (Figure 4). In addition, marked improvement in the patient's mood and anxiety was reported. Post-NFB LORETA imaging confirmed an improvement in electrical dysregulation of previously identified regions (picture not shown). Patient also reported better performance in her academic settings.

\section{Case 3}

27year old law student presented as a self-referral for NFB therapy regarding his cognitive dysfunction; mainly memory. He graduated 
from law school but was unable to study for bar exams due to problems with retaining previously read information. His QEEG brain maps showed mild increase in occipital beta power, temporal delta, and theta power (Figure 5).

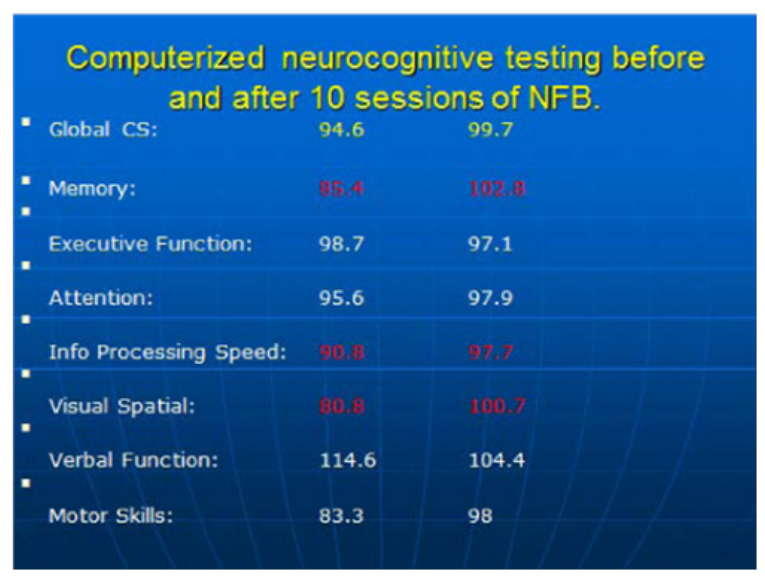

Figure 2 First vertical row shows scores from computerized cognitive testing before NFB. Second vertical row shows scores after 10 sessions of NFB. Computerized cognitive testing (NeuroTrax Corp, Bellaire TX) shows data based on comparison to age and education matched controls. Expected score is 100 with I standard deviation-15.



Figure 358 year old female-LORETA imaging before NFB shows two areas of electrical dysregulation (in red) including the cingulate cortex and left temporal region.

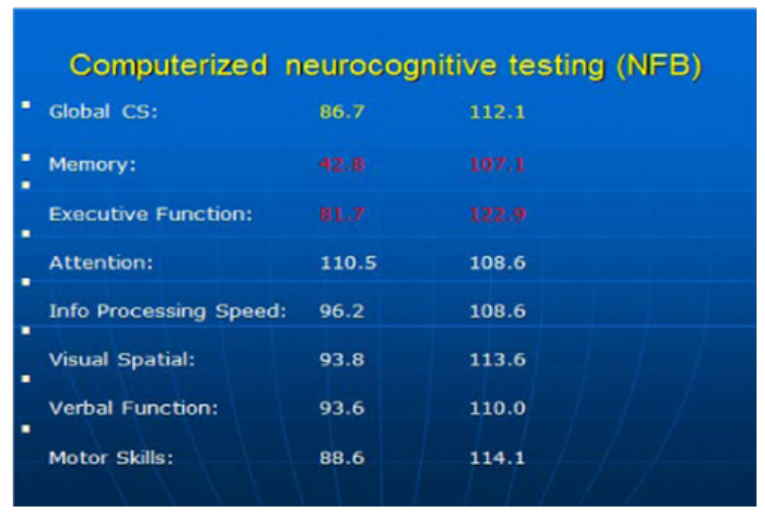

Figure 458 year old female cognitive testing results before (left column) and after (right column) 10 sessions of NFB. Expected score is 100 with I standard deviation- 15.

The neurocognitive testing before NFB showed low memory and information processing speed. After 10 NFB sessions, marked improvement in subjective and objective memory was noted (Figure $6)$.



Figure 5 QEEG maps showed increased bilateral temporal delta and theta power, as well as occipital beta power (left). Marked improvement in the previously dysregulated areas was noted (right) after NFB. Green, yellow, and red colors indicate areas within 0-I, I-2, and 2-3 Standard Deviations (SD), respectively.



Figure 6 Computerized cognitive testing before (left) and after (right) NFB.

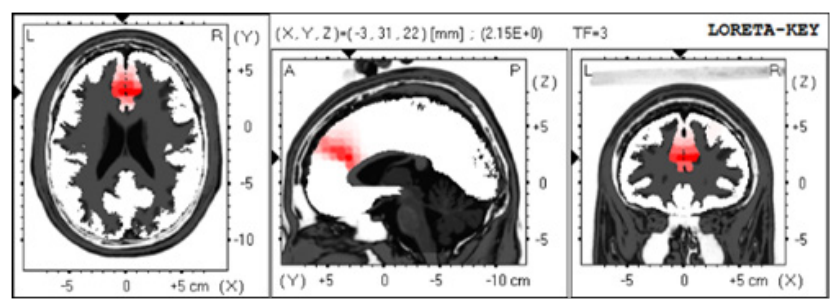

Figure 7a LORETA imaging of 19 year old patient showing area of electrical dysregulation of Anterior Cingulate (AC) region BA 32 (in red).

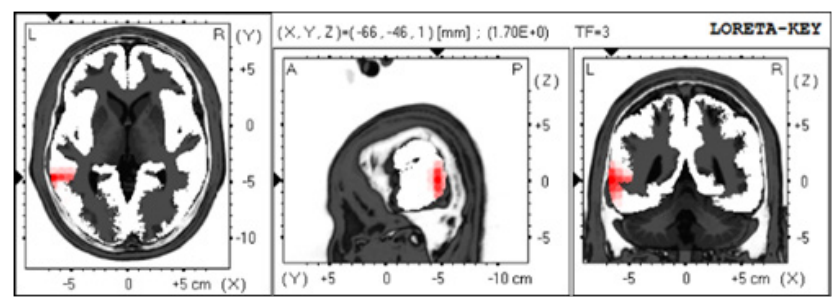

Figure 7b Resolution of AC dysregulation after 10 sessions of NFB.

\section{Case 4}

19year old male was brought for an appointment by his adoptive parents indicating problems with cognition, mostly executive function and information processing speed dysfunction. Initial QEEG revealed frontal increase in delta power however LORETA (Figure 
7A) (Figure 8A) showed anterior cingulate electrical dysregulation. Before initiation of NFB, the neurocognitive testing was completed in March, 2013; results showed low cognitive score-Global Cognitive Score 80.2 with low executive function 87.2 , and a very low information processing speed of 52.1. After the first 10 sessions of NFB, his executive function improved to 89.9 , and with each round of ten NFB sessions, repeated cognitive testing was giving better results with executive function (subsequently 92 and 97; (Figure 9). In addition to improvement of LORETA (Figure 7B, 7C \& 7D) electrical dysregulation as well as QEEG findings (Figure 8B, 8C \& 8D) and cognitive testing, his parents noted a tremendous improvement with social and cognitive performance.



Figure 7c Resolution of AC dysregulation after 20 sessions of NFB.

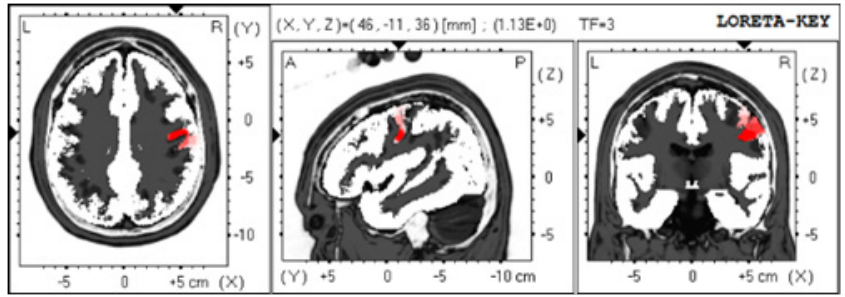

Figure 7d Resolution of AC dysregulation after 30 sessions of NFB.

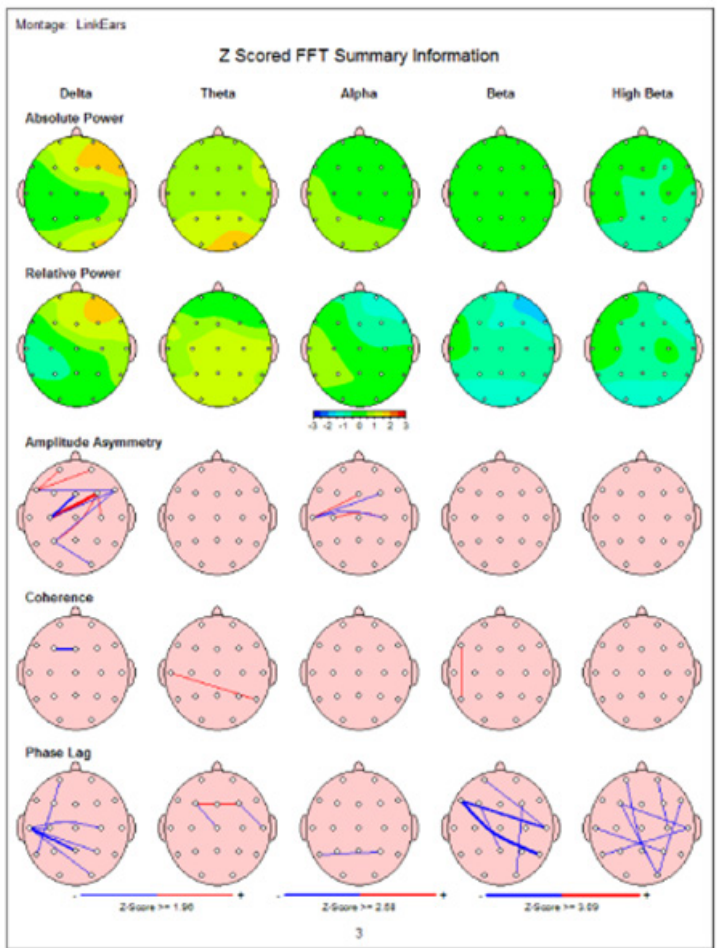

Figure 8a QEEG of 19 year old with cognitive problems before initiation of NFB and after each round of 10 sessions of NFB. QEEG before NFBnoticeable area of frontal increase in delta power.Green, yellow, and red colors indicate areas within $0-\mathrm{I}, \mathrm{I}-2$, and 2-3 Standard Deviations (SD), respectively.

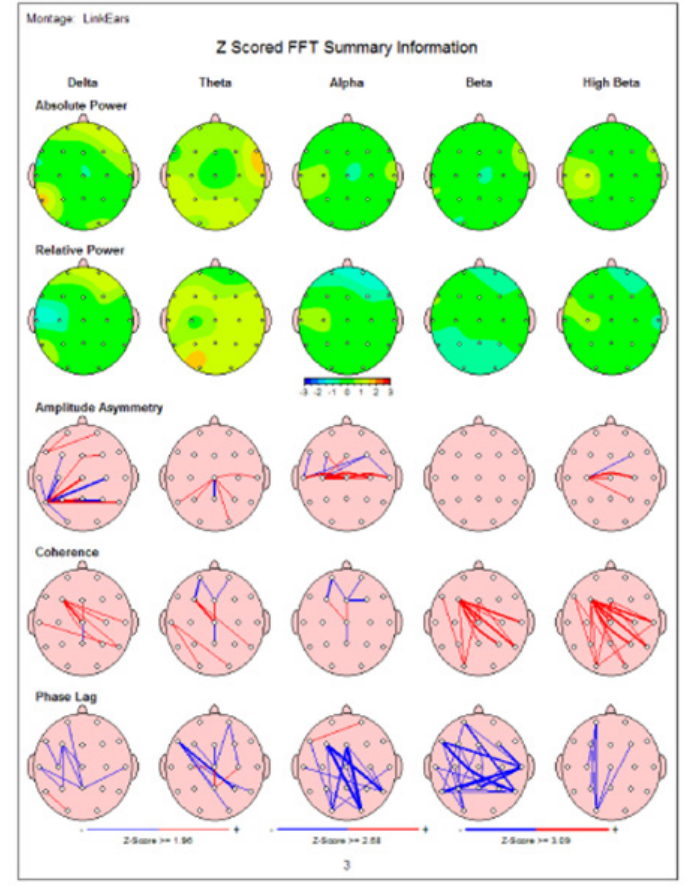

Figure 8b QEEG after 10 sessions of NFB-an improvement in previously over expressed frontal delta power is recorded.

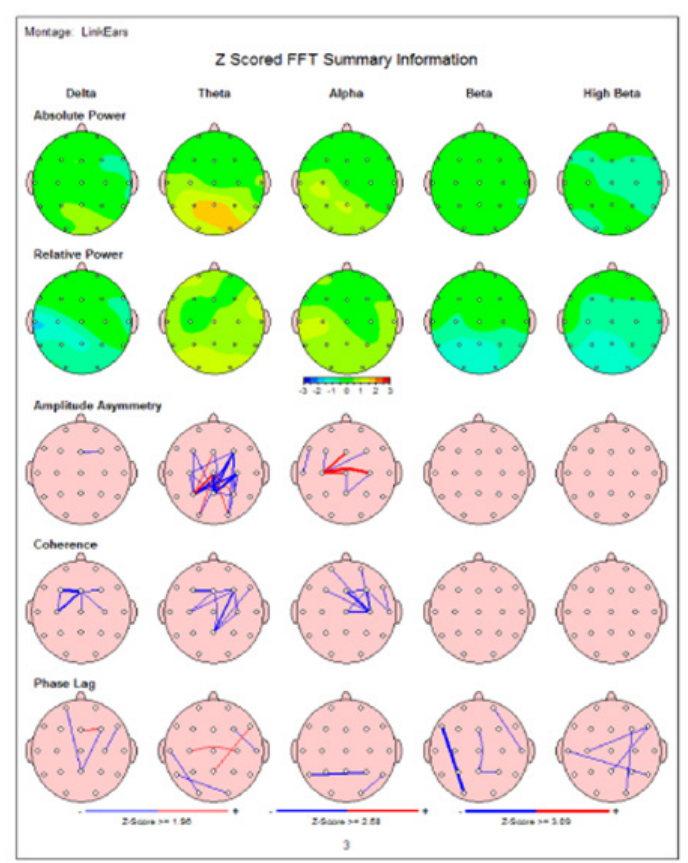

Figure 8C QEEG after 20 NFB sessions-continuous improvements in frontal delta power is noted.

\section{Dementia Cases}

Case 5: 68-year-old female (retired accountant) complained of 1-2years of history of progressive forgetfulness. Patient came to my office for a second opinion with her husband. She was previously seen 
by another neurologist and neuropsychologist, and was diagnosed with early stage of dementia (likely mild AD). She was recommended to start treatment with Aricept. However, the patient was reluctant to initiation of pharmacotherapy. Initial computerized neurocognitive evaluation with NeuroTrax (Bellaire, TX) showed mild impairment of short-term memory-score 85.4 (expected 100), with no major involvement of other cognitive functions (Figure 10).

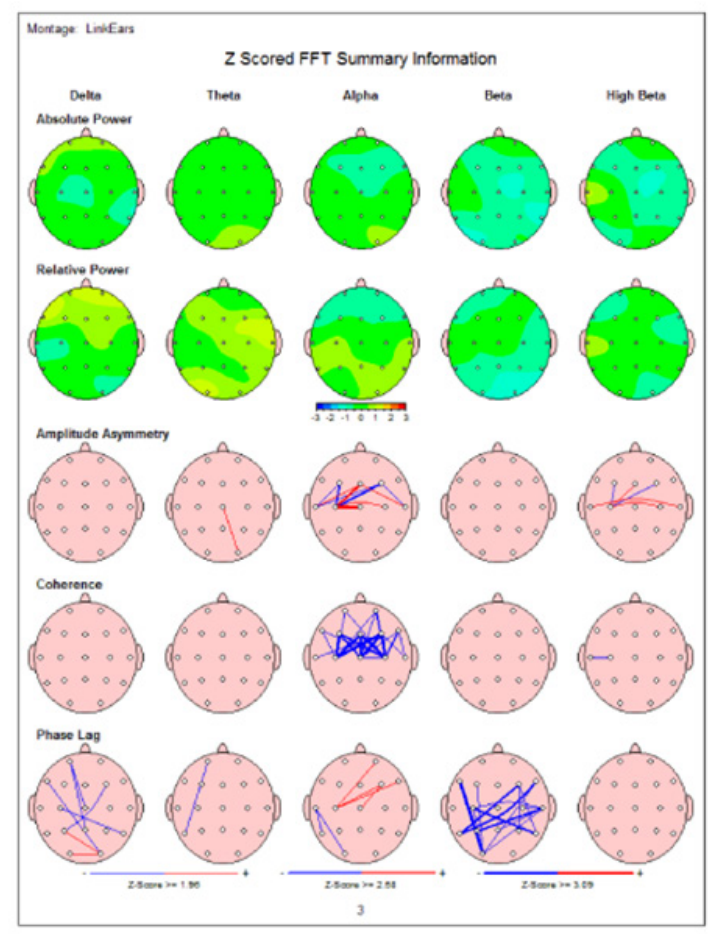

Figure 8D QEEG after 30 sessions-complete normalization of frontal delta over expression is noted.



Figure 9 Executive testing before NFB (March 2013) and after each round of 10 sessions of NFB (in May 2013, October 2013 nd January 2014)-gradual increase in executive function was recorded.

Initial QEEG evaluation showed increase in bilateral temporal delta and global theta power (Figure 11).

Her blood work was unremarkable, including B12 level. Patient was interested in starting alternative therapy, initialized with the standard 1-electrode NFB (Cygnet). Despite several months duration of NFB (with frequency of 2-3times a week), further decline in memory function was noted on follow-up computerized neurocognitive testing, with memory score of 80.1 (Figure 12). At that time, since her symptoms were progressive, the patient decided to start 19-electrode Z-score Low Resolution Electro-magnetic Tomography Analysis (LORETA) NFB (Neuroguide, Applied Neuroscience, Inc.), which became available in our clinic.

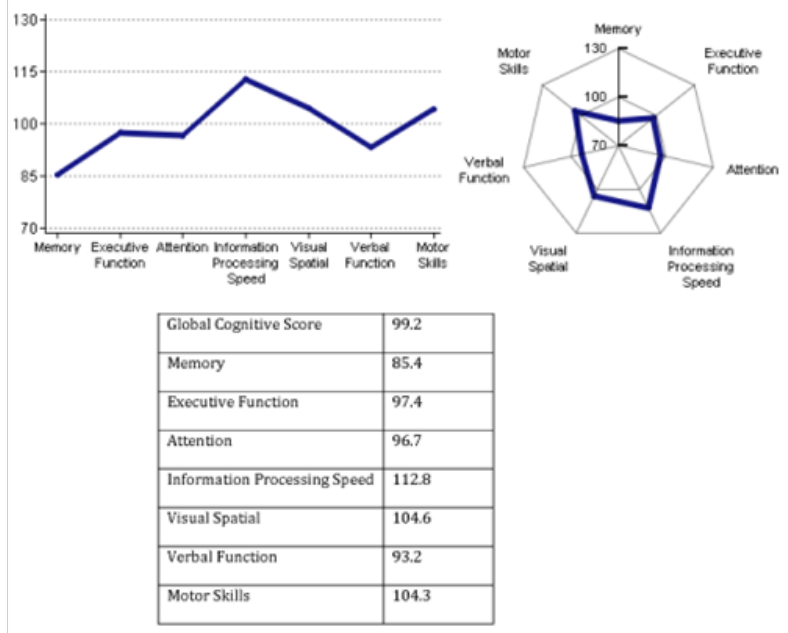

Figure 10 Computerized neurocognitive testing of 68-year-old female. Abnormal is short-term memory score of 85.4 (expected score-100).

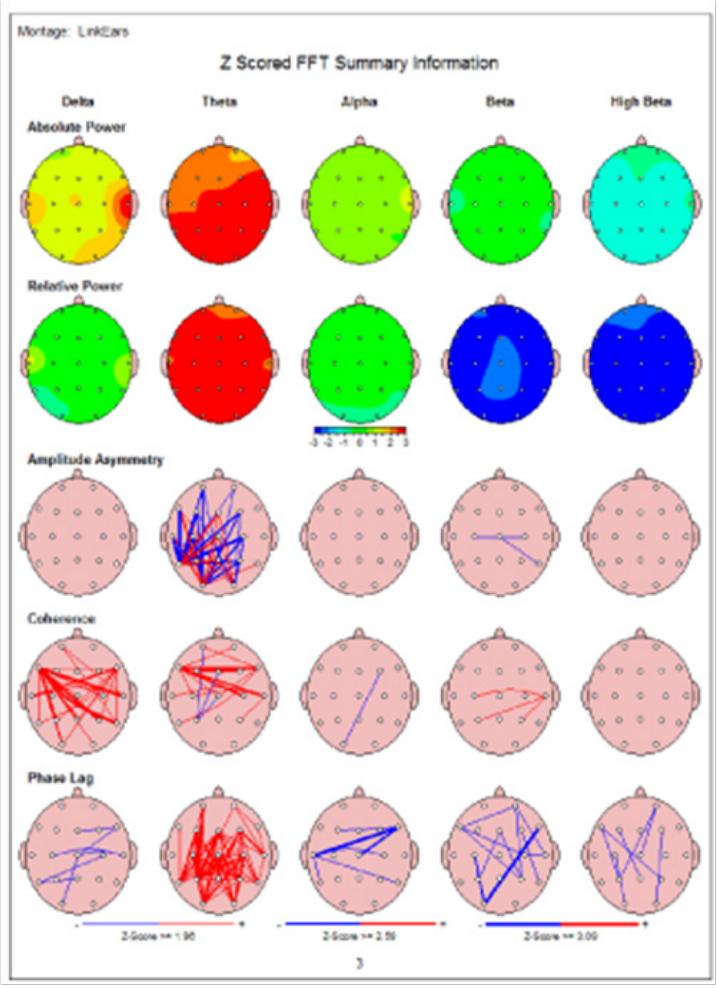

Figure II Shows the summary page of QEEG of 68-year-old patient with memory problems. There is evidence of increased bilateral temporal delta and global theta power (Neuroguide, Applied Neuroscience, Inc.). Green, yellow, and red colors indicate areas within 0-I, I-2, and 2-3 Standard Deviations $(\mathrm{SD})$, respectively.

Antipsychotic drugs can cause a wide range of potential adverse effects. ${ }^{19}$ Adverse effects of antipsychotics are often neither diagnosed nor treated..$^{43}$ In his audit, Cleary $\mathrm{A}^{43}$ investigated current practice within a rural mental health service area on the monitoring and 
documentation of side effects of antipsychotic depot medication. According to the audit, side effects were not recorded in $72 \%$ of cases. Locally developed checklists scales were used in $25 \%$ of the case notes examined, this percentage is low when considering the popularity of using an evidenced-based tool in practice, for example, the Liverpool University Neuroleptic Side Effect Rating Scale (LUNSERS). ${ }^{25} \mathrm{He}$ concluded that Collaborative practice with feedback from service users is essential in service improvement and care delivery. ${ }^{43}$

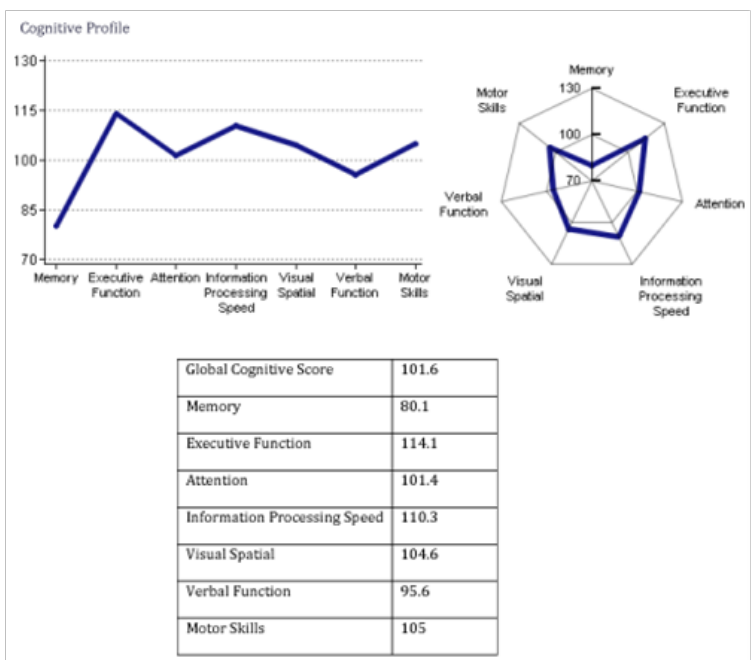

Figure 12 NeuroTrax testing recorded after completion of I-electrode standard NFB therapy. Notice further decline of memory-score (80.I).

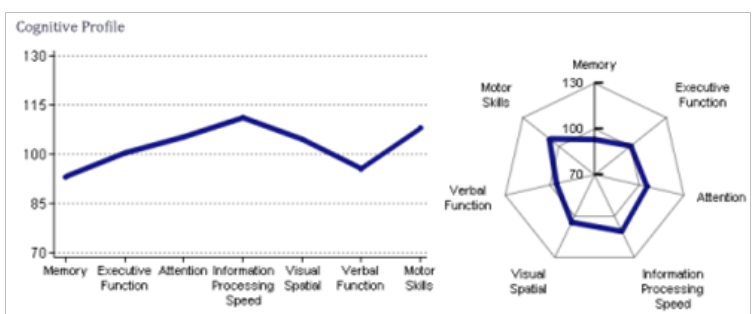

\begin{tabular}{|l|l|}
\hline Global Cognitive Score & 102.6 \\
\hline Memory & 93.2 \\
\hline Executive Function & 100.4 \\
\hline Attention & 105.3 \\
\hline Information Processing Speed & 111.1 \\
\hline Visual Spatial & 104.6 \\
\hline Verbal Function & 95.6 \\
\hline Motor Skills & 107.9 \\
\hline
\end{tabular}

Figure 13 Follow-up Neurotrax testing after completion of 10 sessions of Z-score surface/LORETA NFB showed improved memory score of 93.2.

\section{Case 6}

Seventy eight year old female with three years of mild cognitive problems including short term memory was seen in my clinic for consultation. This patient was not interested in medications but instead initiated NFB. Patient was also evaluated by neuropsychologist and diagnosed with dementia-likely AD. MRI of the brain showed mild chronic ischemic changes nonspecific in character. Computerized cognitive testing showed low GCS-89.8 with very low memory score-67.3. QEEG showed elevation of temporal theta power which frequently is seen in individuals with cognitive dysfunction. Patient completed 10 NFB sessions and noticed improvement of cognitive function. In addition, NeuroTrax cognitive testing completed 10weeks later confirmed marked cognitive benefit of NFB with improved GCS-
100.1 and memory- 84.8 (Table 1). At the same time an improvement of QEEG findings was also noted.
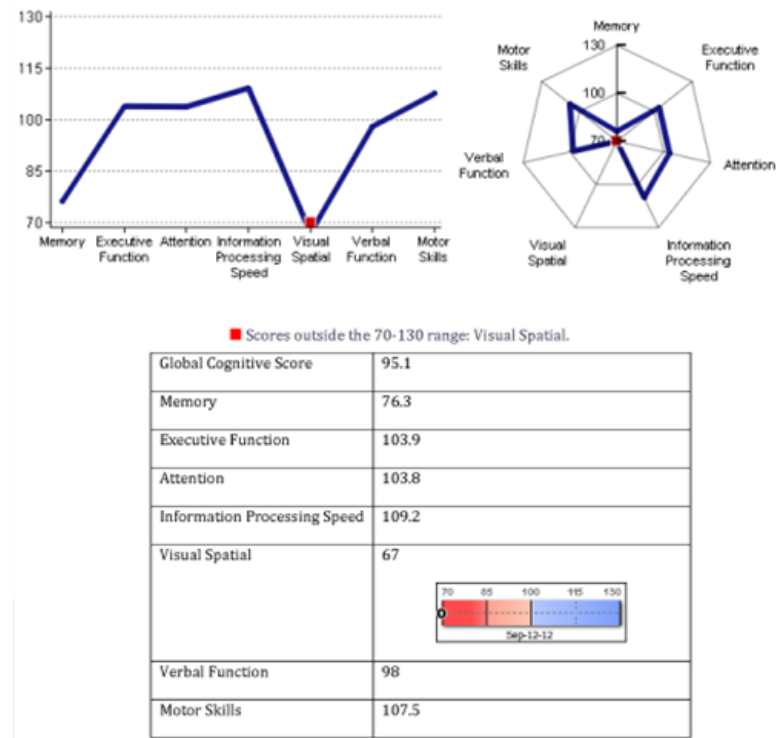

Figure 14 Follow-up neurocognitive testing three and a half months after discontinuation of Z-score 19-electrode NFB. See abnormal memory score-76.3 and visual spatial score of 67.

Table I Computerized cognitive testing before and after I0 NFB sessions

\begin{tabular}{lll}
\hline & Before NFB & After NFB \\
\hline Global Cognitive Score & 89.8 & 100.1 \\
Memory & 67.3 & 84.8 \\
Executive Function & 86.6 & 105.6 \\
Attention & 96.9 & 102.7 \\
Information Processing Speed & 108.6 & 111.5 \\
Visual Spatial & 90.1 & 90.1 \\
Verbal Function & 89.8 & 100.3 \\
Motor Skills & 89.6 & 106 \\
\hline
\end{tabular}

\section{Case 7}

Eighty six year old female was diagnosed with mild AD based on mild cognitive problems with short term memory dysfunction and delusions. Prior CT of the brain testing was reported as WNL. QEEG showed evidence of increased bilateral temporal theta power. Computerized cognitive testing before NFB showed low memory score 74.7 (Table 2). After 10 NFB sessions (2months later) repeated memory score did not change. However additional 10 sessions of NFB (total 20sessions) completed almost three month later gave mild increase in memory-score 77.2. At the same time patients also reported mild improvement in her cognitive functioning. Minimal improvement of QEEG abnormalities was also noted.

\section{Case 8}

Sixty year old retired university professor was referred to me due to mild to moderate cognitive problems due to AD. Her problems started four years earlier. MRI of the brain revealed mild atrophy and chronic ischemic changes. Cognitive testing before NFB initiation was abnormal due to low GCS-81.7 as well as low memory score-45.5 and verbal function-25. LORETA showed area of electrical dysregulation in the right temporal region. Patient completed total of 13 NFB sessions with mild improvement noted subjectively as well as objectively on repeated cognitive testing completed three months later (Table 3). 
Table 2 Comtesting before and afputerized cognitive ter 10 and 20 NFB sessions

\begin{tabular}{llll}
\hline & $\begin{array}{l}\text { Before } \\
\text { NFB }\end{array}$ & $\begin{array}{l}\text { After } \\
\text { 20 }\end{array}$ & NFBs After \\
\hline Global Cognitive Score & 97.7 & 96.2 & 95.5 \\
\hline Memory & 74.7 & 74 & 77.2 \\
\hline Executive Function & 99.8 & 97.3 & 84.7 \\
\hline Attention & 104.9 & 109.8 & 103.4 \\
\hline Information Processing Speed & 116.3 & 105.5 & 94.3 \\
\hline Visual Spatial & 92.8 & 86.2 & 94.3 \\
\hline Verbal Function & 98.9 & 84.7 & 99.5 \\
\hline Motor Skills & 96.5 & 115.8 & 116.6 \\
\hline
\end{tabular}

Table 3 Computerized cognitive testing before and after 10 NFB sessions

\begin{tabular}{lll}
\hline & Before NFB & After 10 NFBs \\
\hline Global Cognitive Score & 81.7 & 84.8 \\
\hline Memory & 45.5 & 53.7 \\
\hline Executive Function & 100.7 & 110.9 \\
\hline Attention & 107 & 113.2 \\
\hline Information Processing Speed & 91.4 & 91 \\
\hline Visual Spatial & 94.8 & 90.3 \\
\hline Verbal Function & 25 & 26.3 \\
\hline Motor Skills & 107.8 & 108.3 \\
\hline
\end{tabular}

\section{Case 9}

Sixty year old female was seen in my clinic due to short term memory problems and depression. MRI of the brain showed mild chronic ischemic changes. Initial cognitive testing was unremarkable except lower executive function-85.2. QEEG showed elevation of frontal beta power which may be seen in individuals with anxiety. Despite completion of 10 NFB sessions (over the period of 4months) her cognitive status continued to deteriorate. This situation resulted in her job termination (secretarial type). She was seen (7months later) by neuropsychologist and was diagnosed with dementia-likely AD.A month later a repeated computerized cognitive testing confirmed deterioration of her cognition (Table 4). At that time this patient was started on Donepezil (Aricept) 10mg daily.

Table 4 Computerized cognitive testing before and after 10 NFB sessions

\begin{tabular}{lll}
\hline NFB Sessions & $\mathbf{0}$ & $\begin{array}{l}\text { I S Sessions ( I 0 } \\
\text { Months Later) }\end{array}$ \\
\hline Global Cognitive Score & 98.6 & 74.7 \\
\hline Memory & 92.3 & 43 \\
\hline Executive Function & 85.2 & 86.5 \\
\hline Attention & 93.9 & 89.3 \\
\hline Information Processing Speed & 99 & 80 \\
\hline Visual Spatial & 121.7 & 80.5 \\
\hline Visual Spatial & 100.8 & 51.4 \\
\hline Motor Skills & 97.3 & 92.4 \\
\hline
\end{tabular}

\section{Discussion}

Our group has already reported evidence of successful cognitive enhancement $t^{2-3}$ with LORETA Z-score NFB. One case report included an individual with Asperger's Syndrome ${ }^{3}$ associated with speech expressive dysfunction; another one described a student with low information processing speed and decreased memory. ${ }^{2}$ Subsequently we have conducted a larger case series report where 35 consecutive patients complaining of cognitive dysfunction were subjected to LORETA Z-score NFB therapy. ${ }^{11-12}$ Before NFB initiation, these patients were evaluated with computerized neurocognitive testing in order to document and confirm any cognitive dysfunction reported in chief complaint.

Many patients underwent brain imaging and laboratory testing to rule out any treatable condition which could have contributed to the patient's symptoms. Electrical imaging with QEEG/LORETA localization was also completed in order to visualize any area of cortical electrical dysregulation which could have been potentially responsible for the patient's symptoms (with specific Brodmann's Areas). The NFB protocol was based either on the patient's symptoms, the area of cortical dysregulation, or both. After 10 sessions of surface/ LORETAZ-score NFB, the computerized cognitive testing and QEEG were repeated in order to see if any increase in cognitive score and/or reduction in QEEG abnormalities were achieved.

In addition, the subjective patient's response was recorded as to whether or not they felt that the therapy was beneficial. 25 patients (71\%) were identified of having an objective improvement (on average 10 points) through cognitive testing. In addition, the subjective cognitive improvement and an objective reduction of QEEG abnormalities with NFB were also achieved in most of the patients. These results were very promising and indicate good effectiveness of LORETA Z-score NFB in cognitive enhancement. These clinical data illustrate high effectiveness of Z-score LORETA NFB therapy in complex neuropsychiatric patients, where an improvement of cognitive domains can be achieved in most of the patients within just 10 treatment sessions.

These cases also illustrate a potentially beneficial application of 19-electrode Z-score NFB in patients suffering from Alzheimer's disease. Given results from this study, it is also likely that LORETA Z-score NFB could provide therapeutic means for improvement of cognitive abilities in AD patients, especially in the early stages of this disease. NFB treatment of this neurodegenerative disorder resulted in temporarily improvement of cognitive function in 4 out of 5 patients (80\%). Unfortunately, since AD is a progressive disorder, further decline of cognitive functions subsequently is likely to occur. Thus, this is not unexpected that after discontinuation of NFB therapy we may see continuation of cognitive decline.

Based on our cases analysis and their performance it seems that for NFB to be effective for cognitive dysfunction more frequent NFB sessions may be required. The only one AD patient who did not benefit from NFB therapy was the one who was unable to visit our center on the weekly basis. Further studies of Z-score surface/ LORETA 19-electrode NFB with a larger number of individuals suffering from $\mathrm{AD}$ may provide additional insights into potential benefits of NFB in this group of patients. I also would recommend a wider implementation of QEEG/LORET a testing as a complementary method in early diagnosis of dementia since the increase in frontal and temporal expression of delta and theta power is frequently seen even in early stages of cognitive dysfunction.

\section{Acknowledgments}

None.

\section{Conflicts of interest}

Author declares there are no conflicts of interest.

\section{Funding}

None. 


\section{References}

1. Koberda JL, Moses A, Koberda P, et al. Clinical advantages of quantitative electroencephalogram (QEEG) -electrical neuroimaging application in general neurology practice. Clin EEG Neurosci. 2013;44(4):273-285.

2. Koberda JL, Moses A, Koberda L, et al. Cognitive Enhancement Using 19-electrode Z-score Neurofeedback. Journal of Neurotherapy. 2012;16(3):224-230.

3. Koberda JL. Autistic Spectrum Disorder (ASD) as a Potential Target of Z-score LORETA Neurofeedback. The Neuroconnection- winter 2012, (ISNR). 2012. p.24.

4. Becerra J, Fernandez T, Roca-Stappung M, et al. Neurofeedback in healthy elderly human subjects with electroencephalographic risk for cognitive disorder. J Alzheimers Dis. 2012;28(2):357-367.

5. Nan W, Rodrigues JP, Ma J, et al. Individual alpha neurofeedback training effect on short term memory. Int J Psychophysiol. 2012;86(1):83-87.

6. Angelakis E, Stathopoulou S, Frymiare JL, et al. EEG neurofeedback: a brief overview and an example of peak alpha frequency training for cognitive enhancement in the elderly. Clin Neuropsychol. 2007;21(1):110-129.
7. Zoefel B, Huster RJ, Herrmann CS. Neurofeedback training of the upper alpha frequency band in EEG improves cognitive performance. Neuroimage. 2011;54(2):1427-1431.

8. Choi SW, Chi SE, Chung SY, et al. Is alpha wave neurofeedback effective with randomized clinical trials in depression? A pilot study. Neuropsychobiology. 2011;63(1):43-51.

9. Robert W, Thatcher. Latest developments in live z-score training: Symptom checklist, phase reset, and LORETA z-score biofeedback. Journal of Neurotherapy. 2013;17(1):69-87.

10. Koberda JL, Koberda P, Bienkiewicz A, et al. Pain Management Using 19-Electrode Z-Score LORETA Neurofeedback. Journal of Neurotherapy. 2013;17(3):179-190.

11. Koberda JL, Moses A, Koberda P, et al. Cognitive Enhancement with LORETA Z-score Neurofeedback. AAPB meeting. 2014.

12. Koberda, JL. Z-score LORETA Neurofeedback as a Potential Therapy in Depression/Anxiety and Cognitive Dysfunction. In: Thatcher, RW \& Lubar JF (Eds.), Z ScoreNeurofeedback: Clinical Applications. Academic Press San Diego, USA. 2014. p.93-114. 